\title{
Supratentorial High Convexity Intradural Extramedullary Cavernous Angioma -Case Report-
}

\author{
Yohtaro SAKAKIBARA, Takashi MATSUMORI, \\ Yoshio TAGUCHI, and Hirotaka KOIZUMI* \\ Division of Neurosurgery and ${ }^{*}$ Department of Pathology, \\ St. Marianna University Yokohama City Seibu Hospital, Yokohama, Kanagawa
}

\begin{abstract}
A 59-year-old man presented with a 2-month history of numbness in the lower left side of the face and upper left extremity. Axial $\mathrm{T}_{1}$-weighted magnetic resonance imaging showed a wedge-shaped mass measuring $3 \times 2.5 \mathrm{~cm}$ in the right frontoparietal high convexity area that was heterogeneously enhanced after administration of gadolinium-diethylenetriaminepenta-acetic acid. Right frontoparietal craniotomy was performed and a bluish soft mass was found under the arachnoid membrane. The mass could be dissected free from the arachnoid membrane and the brain surface. Histological examination revealed the typical findings of cavernous angioma. Cavernous angioma should be considered in the differential diagnosis of supratentorial high convexity intradural extramedullary tumor, especially appearing as a heterogeneously enhanced mass adjacent to the brain parenchyma causing mass effect.
\end{abstract}

Key words: cavernous angioma, intradural extramedullary tumor, supratentorial high convexity, mass sign

\section{Introduction}

Cavernous angioma of the central nervous system usually occurs in the neural parenchyma. ${ }^{6}$ Extramedullary cavernous angiomas are rare, and occur in the intradural extramedullary spaces of the brain or spine such as at the cerebellopontine angle,${ }^{3)}$ on the optic chiasm, ${ }^{2}$ intracavernously, ${ }^{4)}$ in the spinal region, ${ }^{1,5)}$ and others. We present an extremely rare case of cavernous angioma located in the intradural extramedullary space at the supratentorial high convexity.

\section{Case Report}

A 59-year-old man presented with a 2-month history of numbness in the lower left side of the face and upper left extremity. Neurological examination found no abnormalities except for the mild sensory losses. Axial $\mathrm{T}_{1}$-weighted magnetic resonance imaging showed a wedge-shaped, nonspecific intense mass measuring $3 \times 2.5 \mathrm{~cm}$ in the right frontoparietal high convexity area, which was heterogeneously enhanced after administration of gadoliniumdiethylenetriaminepenta-acetic acid (Fig. 1). Both internal and external carotid angiography demonstrated no obvious feeding arteries in the arterial phase, but pooling of
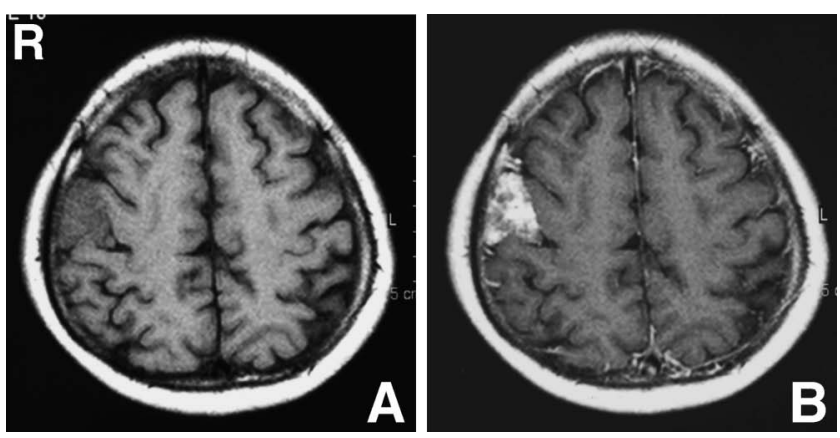

Fig. 1 T $T_{1}$-weighted magnetic resonance images showing the mass as isointense to the gray matter (A) and heterogeneously enhanced with gadolinium-diethylenetriaminepenta-acetic acid (B).

contrast medium in the late venous phase of the internal carotid angiogram (Fig. 2). The preoperative diagnosis was intradural extramedullary mass causing sensory disturbance by simple compression. Right frontoparietal craniotomy was performed. Reflection of the dural flap exposed a bluish soft mass under the arachnoid membrane. The mass could be dissected free from the dura mater, the

Received June 25, 2009; Accepted August 25, 2009

Author's present address: Y. Sakakibara, M.D., D.M.Sc., Department of Neurosurgery, St. Marianna University School of Medicine, Kawasaki, Kanagawa, Japan. 


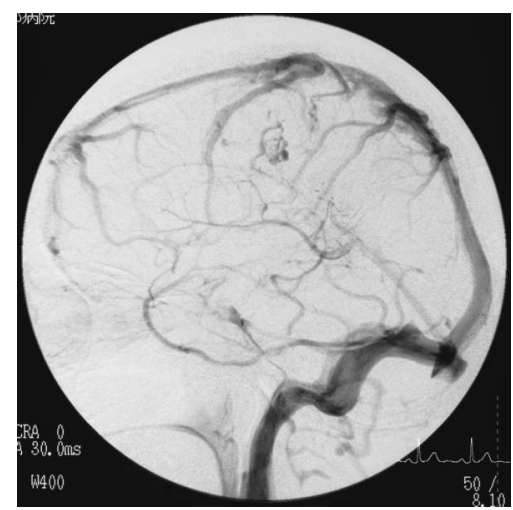

Fig. 2 Right carotid angiogram in the late venous phase revealing pooling of contrast medium.

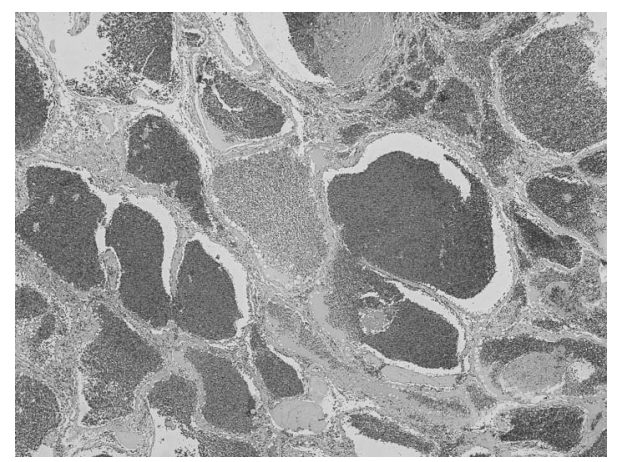

Fig. 3 Photomicrograph of the resected tumor showing multiple dilated thin-walled vascular channels compatible with cavernous angioma. Hematoxylin-eosin stain, $\times 40$.

arachnoid, and the brain surface. No hemosiderin deposits were identified suggesting prior hemorrhage. Eventually the mass was removed en bloc without damaging the underlying brain parenchyma after the draining vein was resected. No obvious feeding arteries were identified. Histological examination revealed irregularly dilated sinusoidal spaces lined with a single cell layer (Fig. 3). The histological diagnosis was cavernous angioma. Postoperatively the complaints of numbness were resolved.

\section{Discussion}

Extramedullary cavernous angiomas seem to cause different symptoms and signs to intramedullary cavernous angiomas. Intramedullary cavernous angiomas present with multiple episodes of intraparenchymal hemorrhages and seizures. ${ }^{6)}$ Extramedullary cavernous angiomas may simply present with the compression effect of the mass, ${ }^{4)}$ and the clinical presentation depends on the location and the size of the lesion. The present patient had hemiparesis, epilepsy, and cranial nerve deficits or numbness. Curious- ly, despite the location in the subarachnoid space, hemorrhage from extramedullary cavernous angioma is extremely rare. ${ }^{6)}$

Generally, cavernous angiomas do not fill with contrast medium on cerebral angiography. ${ }^{4)}$ In the present case, both external and internal carotid angiography demonstrated no apparent feeding arteries. In contrast, delayed retention of contrast medium was seen in the late venous phase. This venous pooling was considered to be a simple draining vein of the cavernous angioma since no dilated medullary veins radiating into an enlarged central vein suggestive of coexistent venous angioma were detected.

Surgical removal of extramedullary cavernous angiomas can be performed safely with low morbidity and mortality rates. ${ }^{1-3,5)}$ However, cavernous angiomas arising from the cavernous sinus are an exception. Intracavernous cavernous angiomas are vascular rich tumors so incision results in vigorous bleeding that is extremely difficult to control. ${ }^{4)}$ In the present case, the cavernous angioma was easily removed en bloc, because of the absence of adhesion to other surrounding structures such as the dural mater, arachnoid membrane, or underlying brain surface.

Intradural extramedullary cavernous angioma at the supratentorial high convexity is a extremely rare vascular anomaly, with no characteristic neuroimaging appearance and clinical presentation. The differential diagnosis of a heterogeneously enhanced mass adjacent to the brain parenchyma should include extramedullary cavernous angioma because symptoms of compression from the cavernous angioma are usually relieved after total excision with low morbidity.

\section{References}

1) Crispino M, Vecchioni S, Galli G, Olivetti L: Spinal intradural extramedullary hemangioma: MRI and neurosurgical findings. Acta Neurochir (Wien) 147: 1195-1198, 2005

2) Iwai Y, Yamanaka K, Nakajima H, Miyaura T: Cavernous angioma of the optic chiasma. Case report. Neurol Med Chir (Tokyo) 39: 617-620, 1999

3) Katayama Y, Tsubokawa T, Yoshida K: Angioma of the cerebellopontine cistern simulating acoustic neurinomas. Surg Neurol 28: 284-286, 1987

4) Meyer F, Lombardi D, Scheithauer B, Nichols D: Extra-axial cavernous hemangiomas involving the dural sinuses. J Neurosurg 73: 187-192, 1990

5) Nozaki K, Inomoto $T$, Takagi $Y$, Hashimoto N: Spinal intradural extramedullary cavernous angioma. Case report. $J$ Neurosurg 99(3 Suppl): 316-319, 2003

6) Porter P, Willinsky R, Harper W, Wallace C: Cerebral cavernous malformations: natural history and prognosis after clinical deterioration with or without hemorrhage. J Neurosurg 87: 190-197, 1997

Address reprint requests to: Yohtaro Sakakibara, M.D., D.M.Sc., Department of Neurosurgery, St. Marianna University School of Medicine, 2-16-1 Sugao, Miyamae-ku, Kawasaki, Kanagawa 216-8511, Japan.

e-mail: y2sakaki@marianna-u.ac.jp 